\title{
Can Mean-Curvature Flow be Modified to be Non-singular?
}

\author{
Michael Kazhdan ${ }^{1}$, Jake Solomon ${ }^{2}$, Mirela Ben-Chen ${ }^{3}$ \\ ${ }^{1}$ Johns Hopkins University ${ }^{2}$ Hebrew University ${ }^{3}$ Stanford University
}
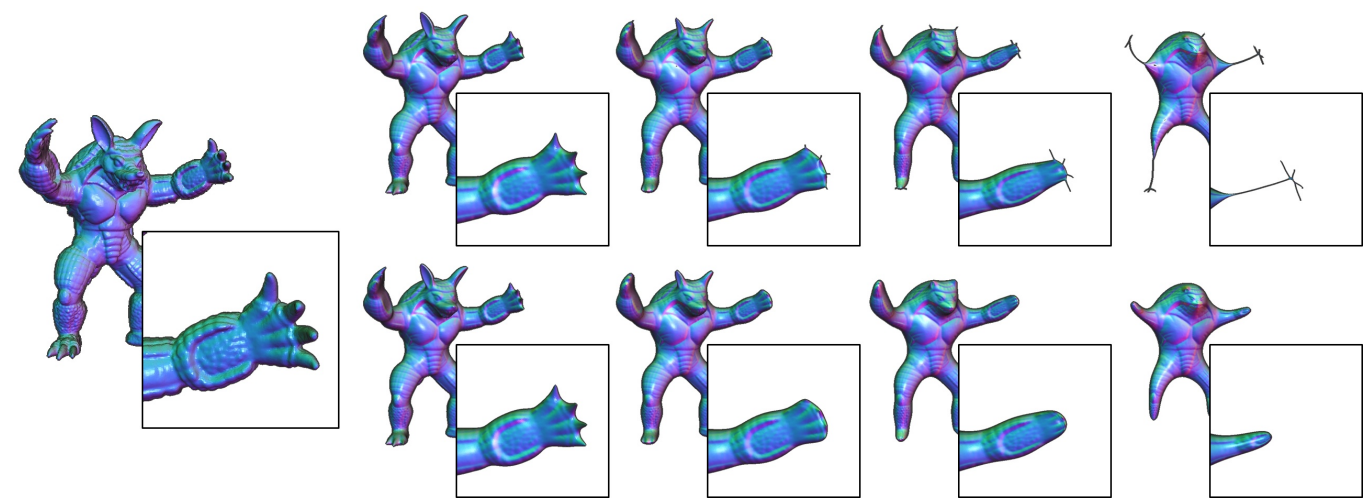

Figure 1: The armadillo man (left) and results of traditional MCF (top) compared to results of our modified MCF (bottom). Both show the evolved surface after 2, 5, 10, and 25 semi-implicit time-steps. The zoom-in on the left hand show that our modified flow avoids the neck pinching singularities that commonly occur in the mean-curvature flow of non-convex regions.

\begin{abstract}
This work considers the question of whether mean-curvature flow can be modified to avoid the formation of singularities. We analyze the finite-elements discretization and demonstrate why the original flow can result in numerical instability due to division by zero. We propose a variation on the flow that removes the numerical instability in the discretization and show that this modification results in a simpler expression for both the discretized and continuous formulations. We discuss the properties of the modified flow and present empirical evidence that not only does it define a stable surface evolution for genus-zero surfaces, but that the evolution converges to a conformal parameterization of the surface onto the sphere.
\end{abstract}

Categories and Subject Descriptors (according to ACM CCS): I.3.5 [Computer Graphics]: Geometric algorithms, languages, and systems-Surface Flow

\section{Introduction}

Mean-Curvature flow is one of the more basic flows that has been used to evolve surface geometry. It can be equivalently formulated as a flow that either (1) minimizes the gradient of the surface embedding or (2) minimizes surface area. As the former, it has played an essential role in the area of mesh fairing, removing noise by smoothing the embedding [Tau95, DMSB99]. As the latter, it has been essential in the study of minimal surfaces [Cho93, PP93].

Despite its pervasiveness, the utility of mean-curvature flow has been restricted by the formation of singularities. As a result, convergence proofs have been limited to a class of simple shapes (e.g. convex surfaces) [Hui84].

In this work, we propose a modification of traditional mean-curvature flow that may address this limitation. We proceed by analyzing the finite-elements discretization of the flow, identify a potential for division-by-zero in the definition of the system matrix, and show that a minor modification to the flow removes the numerical instability, providing simpler expressions for both the discrete and continuous formulations of the flow. An analysis of this flow shows 
that the modification does not change the flow in spherical regions, and it slows down the evolution in cylindrical regions, avoiding the formation of undesirable neck pinches. Though we do not have a proof that it will always happen, we show numerous examples of the flow on highly concave, genus-zero, surfaces, demonstrating that not only is the flow non-singular, but it also converges to a conformal parameterization of the surface onto a sphere.

There exist other important geometric flows, such as Ricci flow [JKLG08] and Willmore flow [BS05], as well as modifications of mean curvature flow [CDR00]. Ricci flow is conformal, guaranteed to evolve a genus-zero surface to a sphere. However, the flow is intrinsic and therefore only provides the metric of the surface and not its embedding. Similar to MCF, Willmore flow is an extrinsic flow. However, unlike our modified MCF, Willmore flow does not converge to a conformal map, and requires higher order surface derivatives, which makes it more difficult to discretize. While it has been shown that Willmore flow converges for surfaces close to a sphere [Sim01], it remains open whether Willmore flow of embedded surfaces can develop singularities in finite time, and counter-examples exist for immersed surfaces [Bla09]. Recently, a conformal Willmore flow has been suggested [CPS11], which is guaranteed to keep the metric conformal to the original surface. However, to date it has only been applied to topological disks. Finally, our modified flow is simpler than flows defined by anisotrpic diffusion as it is isotropic and semi-linear.

An example of the modified mean-curvature flow can be seen in Figure 1. The original Armadillo-Man model is shown on the left. The top row shows the results of traditional mean-curvature flow (with vertices of collapsed regions fixed and taken out of the linear system, following Au et al. [ATC*08]) while the bottom row shows results of the modified flow. Although both approaches smooth the geometry, the concavity of the shape results in singularities when evolving with the traditional flow. For example, the (cylindrical) fingers collapse within 5 iterations, forming neck pinches before they can be merged into the hand. In contrast, the modified flow avoids these types of singularities, slowing down the inward flow of the extremities and allowing them to merge into the appendages before they have an opportunity to collapse. The limit behavior of the flow can be seen in Figure 8, demonstrating that the flow evolves to a conformal parameterization of the model onto a sphere.

\section{Outline}

We review the continuous formulation of mean-curvature flow and its discretization using finite-elements in Section 2. We analyze the numerical stability of the discretization in Section 3 and show how the flow can be modified to avoid potential division-by-zero. We evaluate our approach in Section 4 , showing the flow for several genus-zero surfaces and discussing its properties, and conclude in Section 5.

\section{Review of Mean-Curvature Flow}

In this section, we briefly review mean-curvature flow. We start with the continuous formulation and then describe a semi-implicit, finite-elements discretization. Although both are classic derivations (see e.g. [Man11] for a modern treatment of the continuous formulation, and [Dzi90] for the finite-elements discretization), we repeat them here to help motivate the definition of our modified flow.

\subsection{Continuous Formulation}

Informally, mean-curvature flow can be thought of as a flow that pushes a point on a surface towards the average position of its neighbors. As with image filtering (when replacing a pixel's value by the average value of its neighbors) this flow has the effect of smoothing out the geometry.

Definition (MCF): Let $M$ be a two dimensional manifold, let $\Phi_{t}: M \rightarrow \mathbb{R}^{3}$ be a smooth family of immersions, and let $g_{t}(\cdot, \cdot)$ be the metric induced by the immersion at time $t$. We say that $\Phi_{t}$ is a solution to the mean-curvature flow if:

$$
\frac{\partial \Phi_{t}}{\partial t}=\Delta_{t} \Phi_{t}
$$

where $\Delta_{t}$ is the Laplace-Beltrami operator defined with respect to the metric $g_{t}$.

\subsection{Finite Elements Discretization}

To model the flow in practice, we need to discretize the differential equation. We transform the continuous system of equations into a finite-dimensional system by choosing a function basis $\left\{B_{1}, \ldots, B_{N}\right\}: M \rightarrow \mathbb{R}$. Using this basis, we represent the map at time $t$ by the coefficient vector $\vec{x}(t)=\left\{x_{1}(t), \ldots, x_{N}(t)\right\} \subset \mathbb{R}^{3}$ so that:

$$
\Phi_{t}(p)=\sum_{i=1}^{N} x_{i}(t) B_{i}(p) .
$$

Although we cannot solve Equation 1 exactly, since the solution may not be in the span of the $\left\{B_{i}\right\}$, we can solve it in a least-squares sense using the Galerkin formulation:

$$
\int_{M}\left(\frac{\partial \Phi_{t}}{\partial t} \cdot B_{i}\right) d \mu_{t}=\int_{M}\left(\Delta_{t} \Phi_{t} \cdot B_{i}\right) d \mu_{t} \quad \forall 1 \leq i \leq N
$$

with $d \mu_{t}$ the volume form induced by the metric $g_{t}$.

Setting $D^{t}$ and $L^{t}$ to be the mass and stiffness matrices of the embedding at time $t:^{\dagger}$

$$
D_{i j}^{t}=\int_{M}\left(B_{i} \cdot B_{j}\right) d \mu_{t} \quad L_{i j}^{t}=-\int_{M} g_{t}\left(\nabla_{t} B_{i}, \nabla_{t} B_{j}\right) d \mu_{t}
$$

$\lceil$ We implicitly assume that either $M$ is water-tight or that its boundary is fixed throughout the course of the flow. 
a semi-implicit time discretization defines the linear system relating coefficients at time $t+\delta$ to coefficients at time $t$ :

$$
\left(D^{t}-\delta L^{t}\right) \vec{x}(t+\delta)=D^{t} \vec{x}(t) .
$$

In the above equations, $\nabla_{t}$ denotes the gradient operator with respect to the metric $g_{t}$.

\section{Modifying the Flow}

We begin this section by considering how numerical instabilities can arise with traditional mean-curvature flow and then propose a modified flow that resolves this problem.

\subsection{Numerical Instability}

A challenge of using mean-curvature flow becomes apparent when we compute the coefficients of the matrix $D^{t}-\delta L^{t}$ by integrating with respect to the metric defined by the original embedding, $\Phi_{0}$, rather than the current embedding, $\Phi_{t}$.

To this end, we consider how the geometry is stretched over the course of the flow, characterized by the endomorphism $g_{0}^{-1} \cdot g_{t}{ }^{*}$ This operator is self-adjoint with respect to both $g_{0}$ and $g_{t}$. Its eigenvectors, $v_{1}$ and $v_{2}$, define the principal directions of stretch (orthogonal with respect to both $g_{0}$ and $g_{t}$ ) and its eigenvalues, $\lambda_{1}^{2}$ and $\lambda_{2}^{2}$, define the (squares of the) magnitudes of stretch along these directions.

Note that both the stretch directions, $v_{i}$, and the stretch factors, $\lambda_{i}$, depend on the time parameter $t$. However, we omit it in our notation for simplicity.

\section{The Mass Matrix}

Using the chain rule, we obtain an expression for the $(i, j)$-th coefficients of the mass matrix as:

$$
D_{i j}^{t}=\int_{M} B_{i} \cdot B_{j} \cdot \sqrt{\left|g_{0}^{-1} g_{t}\right|} d \mu_{0},
$$

where $\sqrt{\left|g_{0}^{-1} g_{t}\right|}$ gives the ratio of area elements. Since meancurvature flow is area minimizing, the values of $\left|g_{0}^{-1} g_{t}\right|$ tend to be small so the computation of $D_{i j}^{t}$ is numerically stable.

\section{The Stiffness Matrix}

The situation gets more complicated when computing the stiffness matrix. The challenge here is due to the fact that, as area shrinks, the corresponding derivatives grow. As a result, since mean-curvature flow is area minimizing, there is potential for the Laplacian to blow up.

To make this explicit, we decompose the gradient in the integrand of $L_{i j}^{t}$ into orthogonal components along the principal directions of stretch, $v_{1}$ and $v_{2}$. Then, using the fact that

\footnotetext{
$\ddagger$ Here we identify $g_{t}$ with the map from the tangent space to its dual so that the map $g_{0}^{-1} \cdot g_{t}$ is a well-defined map from the tangent space to itself.
}

shrinking the domain of a function by a factor of $\lambda$ scales its derivative by the same factor, the expression for the $(i, j)$-th coefficient of the stiffness matrix becomes:

$$
\begin{aligned}
L_{i j}^{t} & =-\int_{M} \sum_{k, l=1}^{2} \frac{\partial B_{i}}{\partial v_{k}} \frac{\partial B_{j}}{\partial v_{l}} g_{t}^{k, l} \sqrt{\left|g_{0}^{-1} g_{t}\right|} d \mu_{0} \\
& =-\int_{M} \sum_{k=1}^{2} \frac{\partial B_{i}}{\partial v_{k}} \frac{\partial B_{j}}{\partial v_{k}} \frac{1}{g_{t}\left(v_{k}, v_{k}\right)} \lambda_{1} \lambda_{2} d \mu_{0} \\
& =-\int_{M} \sum_{k=1}^{2} \frac{\partial B_{i}}{\partial v_{k}} \frac{\partial B_{j}}{\partial v_{k}} \frac{1}{g_{0}\left(v_{k}, v_{k}\right)} \frac{\lambda_{1} \lambda_{2}}{\lambda_{k} \lambda_{k}} d \mu_{0}
\end{aligned}
$$

where $g_{t}^{k, l}$ is the $(k, l)$-th coefficient of the inverse of the $2 \times 2$ matrix whose $(i, j)$-th entry is $g_{t}\left(v_{i}, v_{j}\right)$.

Examining Equation 2, we observe that while the value $\frac{\partial B_{i}}{\partial v_{k}} \frac{\partial B_{j}}{\partial v_{k}} \frac{1}{g_{0}\left(v_{k}, v_{k}\right)}$ is stable, as it only depends on the values of the partial derivatives of the $B_{i}$ along directions that are unit-length under the initial metric, $g_{0}$, the stretch ratios $\lambda_{1} \lambda_{2} / \lambda_{2} \lambda_{2}=\lambda_{1} / \lambda_{2}$ and $\lambda_{1} \lambda_{2} / \lambda_{1} \lambda_{1}=\lambda_{2} / \lambda_{1}$ might not be. In particular, as the stretching becomes more anisotropic (i.e. the mapping $\Phi_{t}$ becomes less and less conformal with respect to the metric $g_{0}$ ) one of the two ratios will tend to infinity and the integrand will blow up.

\subsection{Conformalizing the Metric}

Since the instability of the stiffness matrix is the result of anisotropic stretching, we address this problem by replacing the metric $g_{t}$ in the computation of the system coefficients by the closest metric that is conformal to $g_{0}$ :

$$
\tilde{g}_{t}=\sqrt{\left|g_{0}^{-1} g_{t}\right|} g_{0}
$$

\section{FEM Discretization}

For the finite-elements discretization, the implementation of this modification is trivial. Instead of requiring that the stiffness matrix be computed anew at each time-step (as is required by traditional mean-curvature flow), the modified flow simply re-uses the stiffness matrix from time $t=0$.

Specifically, since the conformalized metric $\tilde{g}_{t}$ has the same determinant as the old metric $g_{t}$, the coefficients of the mass matrix are the same regardless of which of the two metrics we use. However, because the two eigenvalues of the conformalized metric are equal, $\tilde{\lambda}_{1}=\tilde{\lambda}_{2}$, the coefficients of the modified stiffness matrix become:

$$
\begin{aligned}
\tilde{L}_{i j}^{t} & =-\int_{M} \sum_{k=1}^{2} \frac{\partial B_{i}}{\partial v_{k}} \frac{\partial B_{j}}{\partial v_{k}} \frac{1}{g_{0}\left(v_{k}, v_{k}\right)} \frac{\tilde{\lambda}_{1} \tilde{\lambda}_{2}}{\tilde{\lambda}_{k} \tilde{\lambda}_{k}} d \mu_{0} \\
& =-\int_{M} \sum_{k, l=1}^{2} \frac{\partial B_{i}}{\partial v_{k}} \frac{\partial B_{j}}{\partial v_{l}} g_{0}^{k, l} d \mu_{0}=L_{i j}^{0},
\end{aligned}
$$

making it independent of time $t$. 


\section{Continuous Formulation}

The conformalization of the metric also results in a simpler continuous formulation, allowing us to replace the PDE in Equation 1 with the following.

Definition (cMCF): Let $(M, h)$ be a two dimensional Riemannian manifold (with metric $h$ ), let $\Phi_{t}: M \rightarrow \mathbb{R}^{3}$ be a smooth family of immersions, and let $g_{t}(\cdot, \cdot)$ be the metric induced by the immersion at time $t$. We say that $\Phi_{t}$ is a solution to the conformalized mean-curvature flow if:

$$
\frac{\partial \Phi_{t}}{\partial t}=\sqrt{\left|g_{t}^{-1} h\right|} \Delta_{h} \Phi_{t}
$$

where $\Delta_{h}$ is the Laplace-Beltrami operator defined with respect to the metric $h$. (For a derivation, see Appendix A.)

Note that if the mapping $\Phi_{t}$ is conformal with respect to $h$, the Laplace-Beltrami operators $\Delta_{h}$ and $\Delta_{t}$ are related by:

$$
\Delta_{t}=\sqrt{\left|g_{t}^{-1} h\right|} \Delta_{h}
$$

and the flows in Equations 1 and 3 are the same.

\section{Results and Discussion}

We begin by examining some examples of the conformalized mean-curvature flow and then proceed to a discussion of its properties. In our discussion, we assume that an initial embedding $\Phi_{0}: M \rightarrow \mathbb{R}^{3}$ is given, and we take $h$ to be the metric induced by this embedding, $h=g_{0}$.

\subsection{Flowing Surfaces}

To better understand how the conformalized flow evolves the embedding, we compare with two other flows. The first is traditional mean-curvature flow, which updates both the mass- and stiffness-matrix at each time-step. The second is the simple heat flow with respect to the metric $h=g_{0}$ that keeps both matrices fixed, and has been proposed for efficient short-term flows for mesh-fairing [DMSB99].

\section{Analytic Flow}

We start by considering three simple examples for which an analytic expression of the flow can be computed. Understanding these simple cases provides some intuition as to why the modified flow might be free of singularities. These examples include the (hyperbolic) catenoid, the (elliptic) sphere, and the (parabolic) infinite cylinder. For each of these geometries and all three flows, the evolved surfaces can be characterized by their radius, $r(t)$, as described in Table 1. (For a derivation, see Appendix B.) Examining this table, we make several observations.

First, for each shape, the derivatives of the three flows at $t=0$ are equal, since all three start by flowing the embedding along the normal direction, with speed equal to the negative of the mean-curvature.

\begin{tabular}{l|l|l|l|} 
& \multicolumn{1}{|c|}{ MCF } & Heat Flow & \multicolumn{1}{c|}{ cMCF } \\
\hline Catenoid & $r(t)=1$ & $r(t)=1$ & $r(t)=1$ \\
\hline Sphere & $r(t)=\sqrt{1-4 t}$ & $r(t)=e^{-2 t}$ & $r(t)=\sqrt{1-4 t}$ \\
\hline Cylinder & $r(t)=\sqrt{1-2 t}$ & $r(t)=e^{-t}$ & $r(t)=1-t$ \\
\hline
\end{tabular}

Table 1: Radii of the catenoid, sphere, and infinite cylinder under the different flows, as a function of time $t$.

Second, the catenoid remains fixed under all three flows since its mean-curvature is everywhere zero.

Third, the heat flow remains stable for all shapes. This is expected since computing the flow is equivalent to repeatedly multiplying by the inverse of the system matrix (giving the characteristic exponent in the radius function) so the long term-behavior can be computed by projecting onto the lower eigenvectors of the system.

Fourth, for the case of the sphere, both MCF and cMCF give the same result. This is because the mean-curvature flow of the embedding of the sphere is conformal, so we have $\tilde{g}_{t}=g_{t}$, and both flows define the same linear system.

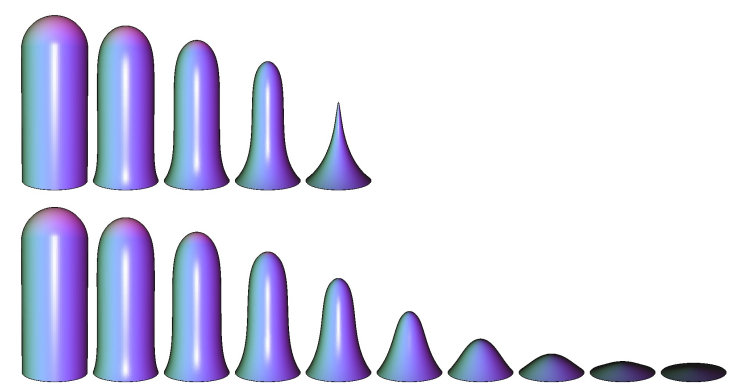

Figure 2: Evolution of an embedding of a rounded cylinder using MCF (top) and the modified flow (bottom). Note that with $c M C F$ the cylinder collapses more slowly, allowing the spherical cap to "catch-up", avoiding the singularity.

Finally, for the cylinder, cMCF slows the rate of shrinking so that the flow towards the cylinder's axis no longer accelerates with time. This is demonstrated in Figure 2, where the first few iterations of MCF and $\mathrm{MMCF}$ are shown. We can see that flowing with $\mathrm{cMCF}$, the cylindrical center collapses more slowly, allowing the spherical top to "catch-up" avoiding the formation of the singularity that appears in MCF.

As we will see next, the same effect allows surface extremities to collapse into the main body without forming neck pinches and, for genus-zero surfaces, evolves into a conformal parameterization of the surface onto a sphere.

\section{Empirical Evaluation}

We ran the modified flow on a number of genus-zero models. For each one, we defined the mass- and stiffness-matrices 
M. Kazhdan, J. Solomon \& M. Ben-Chen / Can Mean-Curvature Flow be Modified to be Non-singular?

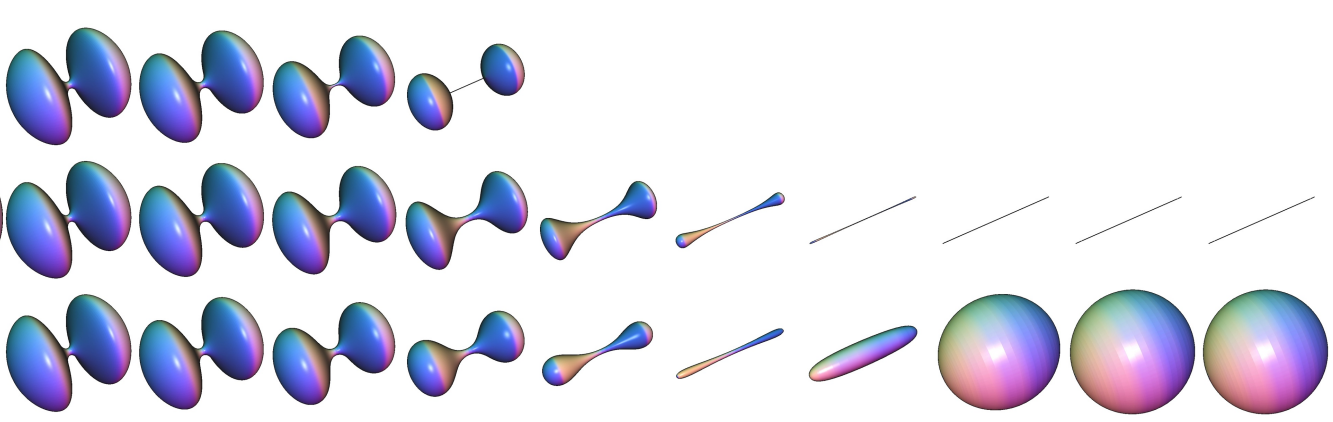

Figure 3: Evolution of a dumbbell model with step-size $\delta=1 \times 10^{-3}$.

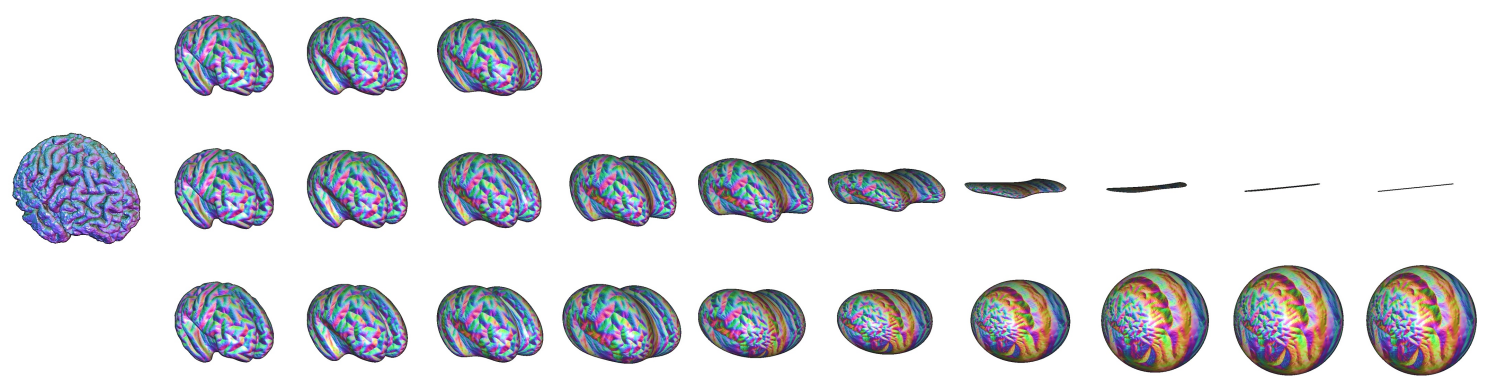

Figure 4: Evolution of brain gray-matter with step-size $\delta=5 \times 10^{-4}$.

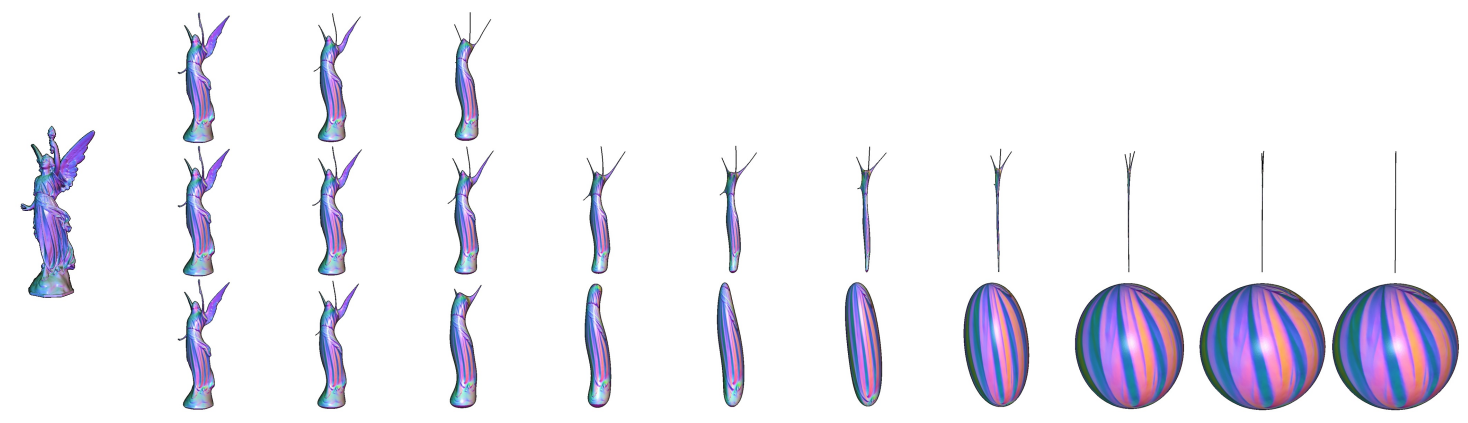

Figure 5: Evolution of the Lucy model with step-size $\delta=5 \times 10^{-4}$.

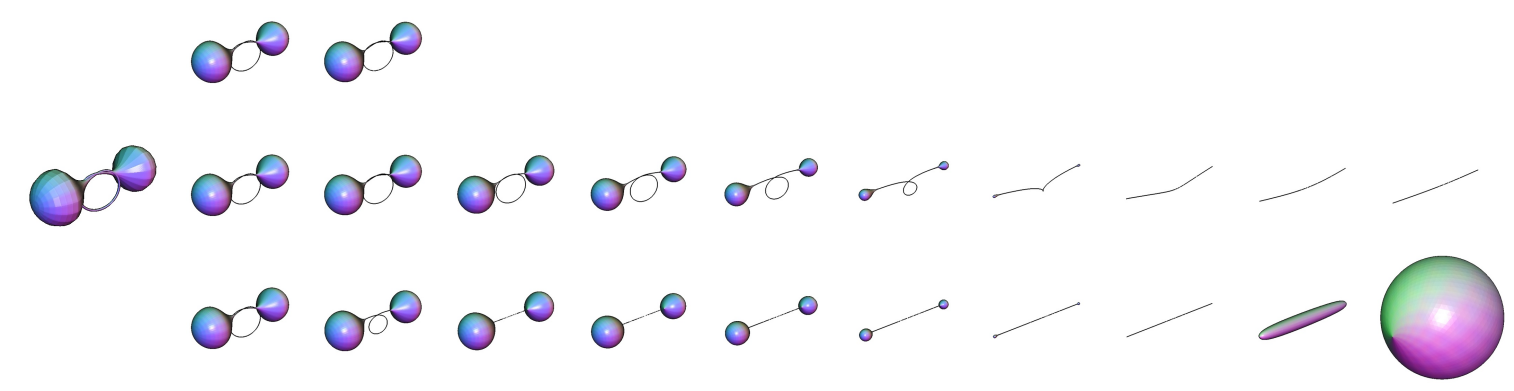

Figure 6: Evolution of a knot with step-size $\delta=5 \times 10^{-4}$. 
using the hat basis [Dzi88]:

$$
\begin{aligned}
& D_{i j}= \begin{cases}\frac{\left|T_{i j}^{1}\right|+\left|T_{i j}^{2}\right|}{\sum_{k \in N(i)}^{12} D_{i k}} & \text { if } j \in N(i)\end{cases} \\
& L_{i j}= \begin{cases}\frac{\cot \beta_{i j}^{1}+\cot \beta_{i j}^{2}}{-\sum_{k \in N(i)}^{2} L_{i k}} & \text { if } j \in N(i) \\
- & \text { if } j=i\end{cases}
\end{aligned}
$$

where $N(i)$ are the indices of the vertices adjacent to vertex $i, T_{i j}^{1}$ and $T_{i j}^{2}$ are the two triangles sharing edge $(i, j)$, and $\beta_{i j}^{1}$ and $\beta_{i j}^{2}$ are the two angles opposite edge $(i, j)$.

We performed the semi-implicit time-stepping using a direct CHOLMOD solver [DH99], running for 512 time-steps, terminating early if numerical instabilities were identified. ${ }^{\S}$ Following [Hui84], we uniformly scaled the map after each step to obtain a surface with unit area. (This is equivalent to reducing the time-step size at larger values of $t$, providing a finer sampling of the flow when the surface evolves faster.)

Our visualizations show the results of traditional meancurvature flow (top), the heat flow (middle), and the modified flow (bottom). They show the original model on the left and the results of the flow for $2^{0}, \ldots, 2^{9}$ time-steps to the right, with per-vertex colors assigned using the original normals.

Figure 3 shows an example of the flow for a dumbbell shape. Because of the concavity at the center, traditional mean-curvature flow quickly creates a singularity (before the 16-th time-step with $\delta=10^{-3}$ ) and the flow cannot proceed. In contrast, the modified flow slows down the collapse near the center, allowing the extremities to collapse more quickly, evolving the embedding into a narrow ellipse which then flows back to a map onto the sphere.

While the heat flow remains stable, it does not tend to evolve towards a smooth shape. (We have validated the longterm behavior of this flow more formally by projecting the embedding function onto the lower-frequency eigenvectors of the Laplace-Beltrami operator.)

Figures 4-6 show challenging examples of flow for highly non-convex shapes including brain gray-matter, the Lucy model, and a twisted dumbbell. Again, we find that MCF quickly creates a singularity and the flow cannot proceed. Similarly, the heat flow does not evolve towards a smooth embedding. It is only using cMCF that the embeddings evolve to maps onto the sphere. (For additional examples of the evolution of embeddings of genus-zero models under $\mathrm{cMCF}$, please see the supplemental material.)

\footnotetext{
$\S$ Numerical instability was defined by failure of CHOLMOD to produce a solution because the system was not positive definite.
}

\subsection{Discussion}

Several questions arise when considering the evolution given by the modified (area-normalized) mean-curvature flow.

\section{Does it converge?}

While this work is motivated by the goal of developing a variation of mean-curvature flow that is non-singular and converges when applied to embeddings of genus-zero surfaces, we have only been able to provide experimental confirmation of this property and leave the proof (or the existence of a counter-example) as an open question. Figure 7 (left) empirically confirms the convergence of the flow for the models in Figure 3-6, giving the magnitude of the difference between successive maps at each iteration.

\section{What does it converge to?}

Looking at Figures 3-6, we observe that modified meancurvature flow appears to always converge to a map onto the sphere. This is confirmed empirically in Figure 7 (right), which plots the variance of the distance of the mesh vertices from its barycenter, as a function of the number of iterations. We see that although the flow may initially make the embedding less spherical, the variance decays in the limit.

\section{How does it converge?}

We are also interested in characterizing the mapping from the original surface to the fixed point of the flow. Comparing the triangulation on the original surface to the triangulation on the limit surface (Figure 8), we see that the limit surface appears to preserve the aspect ratio of the triangles, suggesting that the mapping to the limit surface is conformal.

We confirm this empirically by measuring the quasiconformal error, computed as the area-weighted average of the ratios of the largest to smallest singular values of the map's Jacobian [SSGH01]. For the model in Figure 8, the average quasi-conformal error is 1.034 , which is comparable to the error of 1.033 for the conformal spherical parameterization of Springborn et al. [SSP08]. Visualizations of the errors for both of these maps are shown in the inset on the

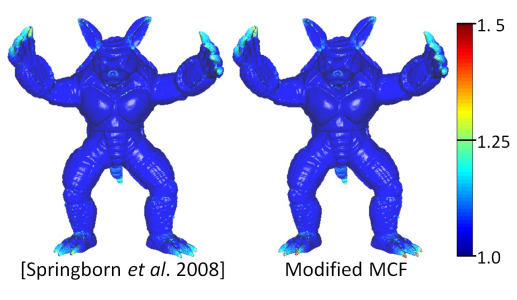
right, highlighting the fact that the quasi-conformal errors are similarly distributed over the surface.

More generally, Figure 7 (middle) shows the plots of these ratios for the shapes in Figures 3-6 as a function of the number of iterations. We see that although the flow is not conformal, since the in-between maps have a high quasi-conformal error, the evolution appears to converge to a conformal map, for all the shapes. 

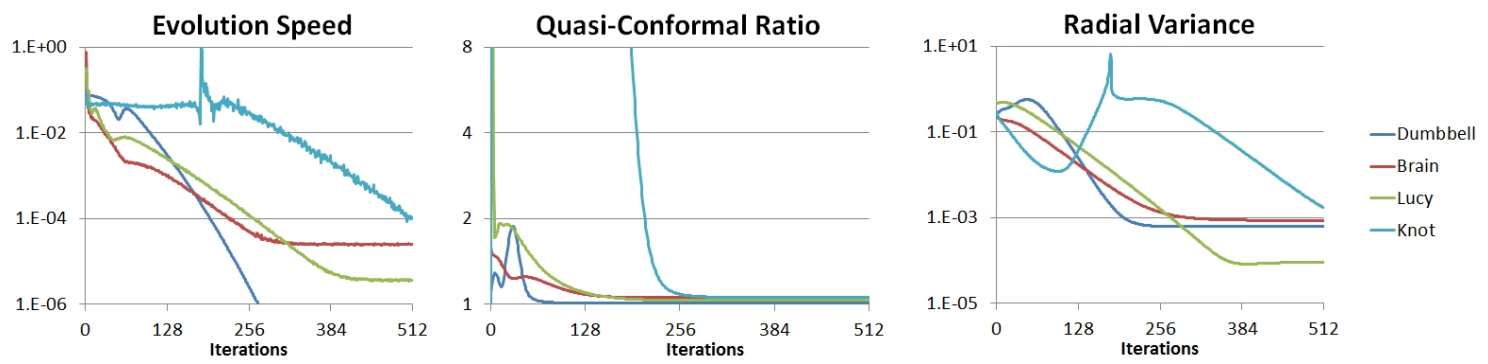

Figure 7: Convergence (left), conformality (middle), and sphericity (right) of cMCF for the models in Figures 3-6.
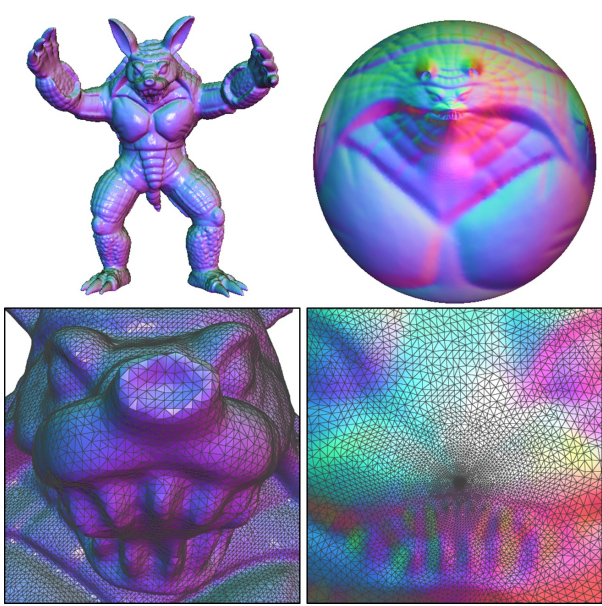

Figure 8: The armadillo-man (left) and the surface obtained as the limit of $c M C F$ (right). Zoom-ins on the triangulation show that the mapping appears to preserve the aspect-ratio of the triangles, suggesting that the mapping is conformal.

\section{Proposition:}

If cMCF converges, than it converges to a map onto the sphere if and only if the limit map is conformal.

\section{Proof:}

$(\Leftarrow)$ If the map $\Phi_{t^{*}}$ is conformal with respect to the metric $h=g_{0}$ then initializing the flow with $\Phi_{0}$ and evolving for time $t^{*}+s$ is equivalent to initializing the flow with $\Phi_{t^{*}}$, setting $h=g_{t^{*}}$, and evolving for time $s$. Thus, evolving the limit map under the modified flow must also result in a uniform scaling of the limit map. Since uniform scaling is itself conformal, this implies that the limit map is uniformly scaled by traditional mean-curvature flow and, since the surface is compact, this implies that the surface is a sphere.

$(\Rightarrow)$ If $\Phi_{t^{*}}$ is a map onto the sphere then we must have $\Delta_{h} \Phi_{t^{*}}=\alpha \sqrt{\left|h^{-1} g_{t^{*}}\right|} \Phi_{t^{*}}$ for some rescaling constant $\alpha$. In particular, this implies that the heat-flow $\partial \Phi_{t} / \partial t=\Delta_{h} \Phi_{t}$ will evolve $\Phi_{t^{*}}$ along directions normal to the surface $\Phi_{t^{*}}(M)$. Thus the function $\Phi_{t^{*}}$, considered as a map from $M$ onto the sphere, is harmonic with respect to the metric $h$ and therefore, by Corollary 1 of [EW76], conformal.

\section{What drives it?}

In this work, the flow was derived by modifying traditional mean-curvature flow. However, one can also interpret the flow as a gradient descent on a non-negative energy. In particular, the flow is a descent on the Dirichlet energy of $\Phi_{t}$ and can be expressed as the sum of a (modified) area energy that drives traditional mean-curvature flow and a conformal energy. (For more details, see Appendix C.)

\section{Other geometries}

While the previous discussion considers embeddings of water-tight, genus-zero surfaces, it is also interesting to consider the behavior of the flow on other geometries. To this end, we have applied our flow both to surfaces with boundaries, and to surfaces with higher genus.

Figures 9 and 10 show the results of our flow for two surfaces with boundaries for which traditional mean-curvature flow generates neck pinches. Though the flow converges, evaluation of the quasi-conformal error shows that the limit map is not conformal. Analyzing the open cylinder, it becomes apparent that stability and conformality cannot be satisfied simultaneously. If the mapping were conformal, then the embedding would be fixed under traditional meancurvature flow, implying that the corresponding surface is minimal. However, since the radius of the cylinder is one and the length of the axis is four, there is no catenoid passing through the two boundaries, and the only minimal surface is the one comprised of two disconnected disks. Thus, the mapping would only be conformal if the flow were to disconnect the surface, which would be impossible without passing through a singularity.

Figure 11 shows the results of our flow on two models that are not simply connected. Though neither flow collapses, they also do not appear to converge. While for higher-genus models the limit map cannot be conformal (as there are no embeddings of compact, non-genus-zero surfaces that are uniformly scaled by mean-curvature flow) it is possible that a limit map exists. However, even if it does, it is not clear that the limit is a 2-manifold. (For example, the embedding of the star appears to converge to a map onto the circle.) 


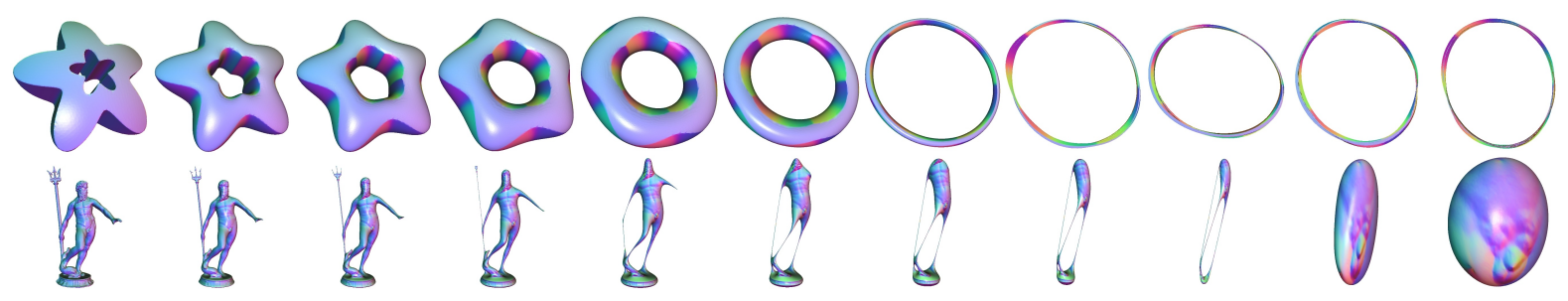

Figure 11: Visualization of cMCF on non-genus-zero models.

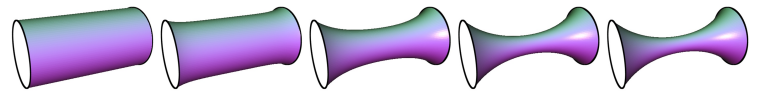

Figure 9: Evolution of an open cylinder under $c M C F$.
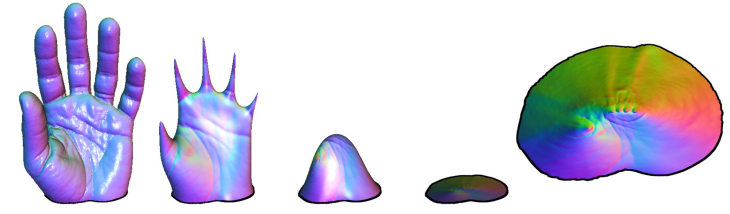

Figure 10: Evolution of an open surface with planar boundary under cMCF (left four images) and a bird's-eye-view of the final mapping (right).

\section{Relationship to 1D Flows}

While traditional mean-curvature flow of embeddings of 2D surfaces in $3 \mathrm{D}$ can form singularities, this is not the case for embeddings of $1 \mathrm{D}$ curves in the plane. In the case of curves, the (uniformly rescaled) flow always converges to a map onto the circle [Gra87]. This agrees with the empirical behavior of our modified mean-curvature flow in that the deformation of the 1D curve is always conformal and the definitions of MCF and $\mathrm{cMCF}$ agree.

Note that, as in the 2D case, (locally) scaling the map by $\alpha$ scales the Laplace-Beltrami operator by $1 / \alpha^{2}$. However, since the $1 \mathrm{D}$ integrals only scale by $\alpha$, the two scaling terms do not cancel out and the discretized Laplace-Beltrami operator does not stay constant.

\section{Conclusion}

In this work, we have considered the problem of singularities that arise in mean-curvature flow when evolving non-convex surfaces. Analyzing the finite-elements discretization that commonly arises in geometry processing, we have associated a potential cause for the formation of singularities with the non-conformality of the flow. We have proposed a modification of the flow that simplifies both the discrete and continuous formulations of the flow. Although we do not have a proof, the work presents empirical evidence that the flow stably evolves genus-zero surfaces, converging to a conformal parameterization of the surface onto the sphere.

Acknowledgements The authors would like to thank William Minicozzi, David Kazhdan, Laurent Younes, Adrian Butscher, Keenan Crane, and Uwe Mayer for invaluable discussions and suggestions.

\section{References}

[ATC*08] Au O., Tai C., Chu H., Cohen-Or D., Lee T.: Skeleton extraction by mesh contraction. ACM Transactions on Graphics (SIGGRAPH '08) 27, 3 (2008). 2

[Bla09] BLATT S.: A singular example for the willmore flow. Analysis 29, 4 (2009), 407-430. 2

[BS05] BOBENKo A. I., SCHRÖDER P.: Discrete willmore flow. In ACM SIGGRAPH 2005 Courses (New York, NY, USA, 2005), SIGGRAPH '05, ACM. 2

[CDR00] Clarenz U., Diewald U., RumpF M.: Anisotropic geometric diffusion in surface processing. Visualization Conference, IEEE 0 (2000), 70. 2

[Cho93] ChорP D.: Computing minimal surfaces via level set curvature flow. Journal of Computational Physics 106 (1993), 77-91. 1

[CPS11] Crane K., Pinkall U., Schröder P.: Spin transformations of discrete surfaces. ACM Trans. Graph. 40 (2011). 2

[DH99] DAVIS T., HAGER W.: Modifying a sparse Cholesky factorization. SIAM Journal on Matrix Analysis and Applications 20 (1999), 606-627. 6

[DMSB99] Desbrun M., Meyer M., Schröder P., BARR A.: Implicit fairing of irregular meshes using diffusion and curvature flow. In ACM SIGGRAPH Conference Proceedings (1999), pp. 317-324. 1, 4

[Dzi88] DzIUK G.: Finite elements for the Beltrami operator on arbitrary surfaces. In Partial Differential Equations and Calculus of Variations, Lecture Notes in Mathematics, vol. 1357. 1988, pp. 142-155. 6

[Dzi90] DZIUK G.: An algorithm for evolutionary surfaces. $\mathrm{Nu}$ merische Mathematik 58, 1 (1990), 603-611. 2

[EW76] EELLS J., Wood J.: Restrictions on harmonic maps of surfaces. Topology 15, 3 (1976), 263-266. 7

[Gra87] GRAYSON M.: The heat equation shrinks embedded plane curves to round points. Journal of Differential Geometry 26 (1987), 285-314. 8 
[Hui84] Huisken G.: Flow by mean curvature of convex surfaces into spheres. Journal of Differential Geometry 20 (1984), 237-266. 1, 6, 10

[JKLG08] JIN M., KIM J., LUO F., GU X.: Discrete surface ricci flow. Visualization and Computer Graphics, IEEE Transactions on 14, 5 (2008), 1030-1043. 2

[Man11] Mantegazza C.: Lecture Notes on Mean Curvature Flow. Birkhauser Verlag, 2011. 2

[PP93] Pinkall U., Polthier K.: Computing discrete minimal surfaces and their conjugates. Experimental Mathematics 2 (1993), 15-36. 1

[Sim01] Simonett G.: The willmore flow for near spheres. Differential and Integral Equations 14 (2001), 1005-1014. 2

[SSGH01] SANDER P., SNyder J., Gortler S., Hoppe H.: Texture mapping progressive meshes. In ACM SIGGRAPH Conference Proceedings (2001), pp. 409-416. 6

[SSP08] SPRINGborn B., Schröder P., PINKall U.: Conformal equivalence of triangle meshes. ACM Transactions on Graphics (SIGGRAPH '08) 27 (2008), 77:1-77:11. 6

[Tau95] TAUBIN G.: A signal processing approach to fair surface design. In ACM SIGGRAPH Conference Proceedings (1995), pp. 351-358. 1

\section{Appendix A: Continuous Formulation}

Although our work has focused on the finite-elements formulation of MCF, the modified flow can also be formulated in a continuous framework.

Claim: cMCF is driven by the PDE:

$$
\frac{\partial \Phi_{t}}{\partial t}=\sqrt{\left|g_{t}^{-1} h\right|} \Delta_{h} \Phi_{t}
$$

Proof: To show this, we choose a test function $B: M \rightarrow \mathbb{R}$ and consider the Galerkin formulation of the PDE:

$$
\int_{M}\left(\frac{\partial \Phi_{t}}{\partial t} \cdot B\right) d \mu_{t}=\int_{M}\left(\sqrt{\left|g_{t}^{-1} h\right|} \Delta_{h} \Phi_{t} \cdot B\right) d \mu_{t} .
$$

Note that, because the metric changes over the course of the evolution, the integrands are expressed with respect to the measure $d \mu_{t}$ not $d \mu_{h}$.

Applying the change-of-coordinates formula, followed by the Divegence Theorem, we get the weak formulation:

$$
\begin{aligned}
\int_{M}\left(\sqrt{\left|h^{-1} g_{t}\right|} \frac{\partial \Phi_{t}}{\partial t} \cdot B\right) d \mu_{h} & =\int_{M}\left(\Delta_{h} \Phi_{t} \cdot B\right) d \mu_{h} \\
& =-\int_{M} h\left(\nabla_{h} \Phi_{t}, \nabla_{h} B\right) d \mu_{h}
\end{aligned}
$$

which gives rise to the discretization with varying massmatrix (lhs) but fixed LB operator (rhs), as in Section 3.

\section{Appendix B: Analytic Flow Solutions}

To better understand the behavior of the flows, we consider three simple surfaces: The (hyperbolic) catenoid, the (elliptic) sphere, and the (parabolic) infinite cylinder. For each, we analyze the evolution of the embedding of the surface under the actions MCF, heat flow, and cMCF.

Our analysis uses the fact that the Laplacian of the embedding is the mean-curvature weighted normal, $\Delta \Phi=-H \vec{N}$.

\section{Catenoid}

Since the catenoid has mean-curvature zero, $H=0$, the Laplacian of the embedding is zero and the surface does not evolve under any of the flows.

\section{Sphere}

Due to the rotational symmetry of the sphere, we know that its evolution under all three flows will have the form $\Phi_{t}(p)=r(t) \cdot \vec{N}(p)$ for some radius function $r(t)$ and normal vector $\vec{N}(p)=p$, so that $\partial \Phi_{t} / \partial t=r^{\prime}(t) \cdot \vec{N}(p)$. Without loss of generality, we take the initial radius to be one.

Traditional MCF Using the fact that the mean-curvature of a sphere of radius $r$ is $2 / r$, we have:

$$
\begin{aligned}
\frac{\partial \Phi_{t}}{\partial t}=\Delta_{t} \Phi_{t}=-\frac{2 \vec{N}}{r} & \Longrightarrow r^{\prime}(t)=-\frac{2}{r(t)} \\
& \Longrightarrow r(t)=\sqrt{1-4 t}
\end{aligned}
$$

Heat Flow For the heat flow, the surface is evolved by always using the Laplacian at time $t=0$ :

$$
\begin{aligned}
\frac{\partial \Phi_{t}}{\partial t}=\Delta_{0} \Phi_{t}=r \cdot \Delta_{0} \Phi_{0}=-2 r \cdot \vec{N} & \Longrightarrow r^{\prime}(t)=-2 r(t) \\
& \Longrightarrow r(t)=e^{-2 t}
\end{aligned}
$$

Modified MCF Using Equation 3, the evolution of the surface under the modified flow can be described by scaling the heat flow by the reciprocal of the area change:

$$
\begin{aligned}
\frac{\partial \Phi_{t}}{\partial t}=\frac{-2 r \cdot \vec{N}}{r^{2}}=-\frac{2 \vec{N}}{r} & \Longrightarrow r^{\prime}(t)=-\frac{2}{r(t)} \\
& \Longrightarrow r(t)=\sqrt{1-4 t}
\end{aligned}
$$

\section{(Infinite) Cylinder}

Due to the translational and rotational symmetries of the cylinder, we know that its embedding will evolve as a constant offset from the original cylinder along the normal, $\Phi_{t}(p)=\Phi_{0}(p)-\vec{N}(p)+r(t) \cdot \vec{N}(p)$ for some radius function $r(t)$, so that $\partial \Phi_{t} / \partial t=r^{\prime}(t) \cdot \vec{N}(p)$. Without loss of generality, we take the initial radius to be one.

Traditional MCF Since the mean-curvature of a cylinder of radius $r$ is $1 / r$, we have:

$$
\begin{aligned}
\frac{\partial \Phi_{t}}{\partial t}=\Delta_{t} \Phi_{t}=-\frac{\vec{N}}{r} & \Longrightarrow r^{\prime}(t)=-\frac{1}{r} \\
& \Longrightarrow r(t)=\sqrt{1-2 t} .
\end{aligned}
$$


Heat Flow For the heat flow, we use the fact that, on a unitradius cylinder, the Laplacian of the normal is equal to the Laplacian of the embedding, $\Delta_{0} \vec{N}=\Delta_{0} \Phi_{0}=-\vec{N}$ :

$$
\begin{aligned}
\frac{\partial \Phi_{t}}{\partial t}=\Delta_{0}\left(\Phi_{0}-\vec{N}+r \cdot \vec{N}\right)=-r \cdot \vec{N} & \Longrightarrow r^{\prime}(t)=-r(t) \\
& \Longrightarrow r(t)=e^{-t} .
\end{aligned}
$$

Modified MCF As above, we use Equation 3 to get:

$$
\begin{aligned}
\frac{\partial \Phi_{t}}{\partial t}=\frac{-r \cdot \vec{N}}{r}=-\vec{N} & \Longrightarrow r^{\prime}(t)=-1 \\
& \Longrightarrow r(t)=1-t .
\end{aligned}
$$

Appendix C: Energy of the Flow

We show that cMCF can be formulated as the gradient flow of an energy that is the sum of a smoothness term, adapted from the energy defining traditional MCF, and a conformal energy. As we will see, this modified energy is just the Dirichlet energy of $\Phi_{t}$ with respect to the metric $h$.

\section{Energies}

Using the Euler-Lagrange formulation, traditional MCF can be defined as the gradient flow of the area functional:

$$
E_{A}\left(\Phi_{t}\right)=\operatorname{Area}\left(\Phi_{t}(M)\right)=\int_{M} \frac{\left|h^{-1} g_{t}\right|}{\sqrt{\left|h^{-1} g_{t}\right|}} d \mu_{h} .
$$

We define a conformal energy by measuring the extent to which $h^{-1} g_{t}$ differs from a scalar multiple of the identity. Specifically, setting $\operatorname{Tr}\left(h^{-1} g_{t} / 2\right) \cdot$ id. to be the scalar multiple of the identity with the same trace as $h^{-1} g_{t}$, we set:

$$
\begin{aligned}
E_{C}\left(\Phi_{t}\right) & =\int_{M}\left(\frac{\left\|h^{-1} g_{t}-\operatorname{Tr}\left(h^{-1} g_{t} / 2\right) \cdot \mathrm{id} .\right\|_{F}^{2}}{\left|h^{-1} g_{t}\right|}\right) d \mu_{t} \\
& =\frac{1}{2} \int_{M} \frac{\operatorname{Tr}^{2}\left(h^{-1} g_{t}\right)-4\left|h^{-1} g_{t}\right|}{\sqrt{\left|h^{-1} g_{t}\right|}} d \mu_{h}
\end{aligned}
$$

where the division by the determinant of $h^{-1} g_{t}$ makes the integrand invariant to uniform scaling of the map $\Phi_{t}$.

\section{Modifying the Energies}

To define the energy driving our modified MCF, we simplify the energies by replacing the geometric mean of the eigenvalues in the denominator, $\sqrt{\left|h^{-1} g_{t}\right|}$, with the arithmetic mean, $\operatorname{Tr}\left(h^{-1} g_{t}\right) / 2$, and sum the (modified) energies. ${ }^{\text {II }}$

If Note that both denominators scale quadratically with $\Phi_{t}$, we have $\operatorname{Tr}\left(h^{-1} g_{t} / 2\right) \leq \sqrt{\left|h^{-1} g_{t}\right|}$, and the two are equal if and only if $\Phi_{t}$ is conformal with respect to the metric $h$.
Replacing the denominators, we get:

$$
\begin{aligned}
& \tilde{E}_{A}\left(\Phi_{t}\right)=2 \int_{M} \frac{\left|h^{-1} g_{t}\right|}{\operatorname{Tr}\left(h^{-1} g_{t}\right)} d \mu_{h} \\
& \tilde{E}_{C}\left(\Phi_{t}\right)=\frac{1}{2} \int_{M} \frac{\operatorname{Tr}^{2}\left(h^{-1} g_{t}\right)-4\left|h^{-1} g_{t}\right|}{\operatorname{Tr}\left(h^{-1} g_{t}\right)} d \mu_{h} .
\end{aligned}
$$

And, taking the sum of the energies, we get:

$$
\tilde{E}\left(\Phi_{t}\right)=\tilde{E}_{A}\left(\Phi_{t}\right)+\tilde{E}_{C}\left(\Phi_{t}\right)=\frac{1}{2} \int_{M} h\left(\nabla_{h} \Phi_{t}, \nabla_{h} \Phi_{t}\right) d \mu_{h} .
$$

That is, we replace the area functional defining traditional MCF with the Dirichlet energy of the map $\Phi_{t}$ with respect to the metric $h$.

Linearizing the energy by considering $\Psi: M \rightarrow \mathbb{R}^{3}$ gives:

$$
\begin{aligned}
\tilde{E}\left(\Phi_{t}+\varepsilon \Psi\right) & =\tilde{E}\left(\Phi_{t}\right)-\varepsilon \int_{M}\left(\Delta_{h} \Phi_{t}\right) \cdot \Psi d \mu_{h}+O\left(\varepsilon^{2}\right) \\
& =\tilde{E}\left(\Phi_{t}\right)-\left\langle\Delta_{h} \Phi_{t}, \Psi\right\rangle_{h}+O\left(\varepsilon^{2}\right) \\
& =\tilde{E}\left(\Phi_{t}\right)-\left\langle\sqrt{\left|g_{t}^{-1} h\right| \Delta_{h}} \Phi_{t}, \Psi\right\rangle_{t}+O\left(\varepsilon^{2}\right)
\end{aligned}
$$

where $\langle\cdot, \cdot\rangle_{t}$ is the inner-product defined on the space of functions on $M$ by the metric $g_{t}$ :

$$
\langle f, g\rangle_{t}=\int_{M}(f \cdot g) d \mu_{t} .
$$

Thus, the gradient of the energy $\tilde{E}$, defined with respect to the inner-product $\langle\cdot, \cdot\rangle_{t}$, is $-\sqrt{\left|g_{t}^{-1} h\right|} \Delta_{h} \Phi_{t}$.

\section{Relationship to Heat Flow}

Note that the heat flow:

$$
\frac{\partial \Phi_{t}}{\partial t}=\Delta_{h} \Phi_{t}
$$

can also be defined as a gradient flow on the Dirichlet energy of $\Phi_{t}$ with respect to the metric $h$. However, as demonstrated in Section 4, heat flow and cMCF evolve the surfaces in different ways. This is because defining the energy gradient requires choosing an inner-product on the space of functions on $M$. For heat flow, the inner-product is defined by the initial metric $h$ giving $\langle\cdot, \cdot\rangle_{h}$ while for cMCF it is defined by the metric $g_{t}$ giving $\langle\cdot, \cdot\rangle_{t}$.

In particular, though both (un-normalized) flows evolve towards the same critical point, (the constant map taking all points in $M$ to a single point in 3D), they converge to this function in different ways. Our modified MCF appears to converge to the constant function "as a sphere" ( [Hui84]) while the heat flow does not. 